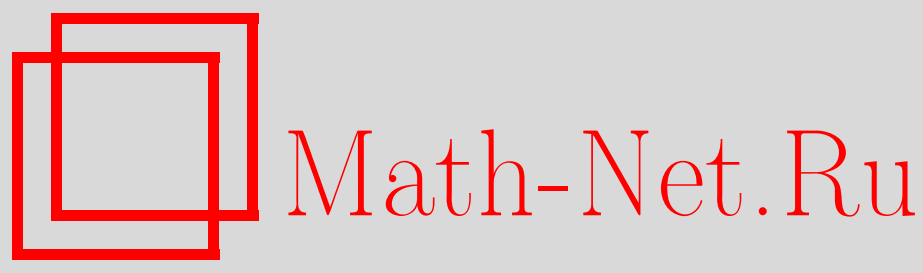

А. И. Олемской, О. В. Ющенко, А. Ю. Бадалян, Статистическая теория поля неаддитивной системы, ТМФ, 2013, том 174, номер 3, 444-466

DOI: https://doi.org/10.4213/tmf8408

Использование Общероссийского математического портала Math-Net.Ru подразумевает, что вы прочитали и согласны с пользовательским соглашением http://www. mathnet.ru/rus/agreement

Параметры загрузки:

IP: 3.95 .254 .165

26 апреля 2023 г., 16:47:00

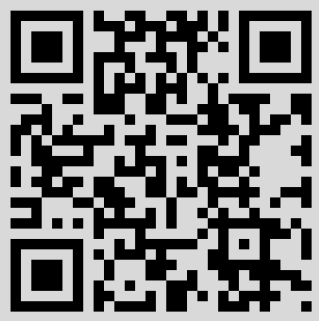




\section{СТАТИСТИЧЕСКАЯ ТЕОРИЯ ПОЛЯ НЕАДДИТИВНОЙ СИСТЕМЫ}

На основе квантово-полевых методов развита статистическая теория сложных систем, термодинамические потенциалы которых не обладают свойством аддитивности. В рамках метода Мартина-Сиггиа-Роуза найден эффективный лагранжиан системы, исходя из которого определены уравнения эволюции наиболее вероятных значений параметра порядка и амплитуды его флуктуаций. Показано, что деформация статистического распределения не изменяет эти уравнения, тогда как вероятность реализации различных фазовых траекторий существенно зависит от параметра неаддитивности. Найден производящий функционал неаддитивной системы и установлена его связь с корреляторами, введена пара аддитивных производящих функционалов, разложение которых дает набор многоточечных функций Грина и их собственно-энергетических частей. Найдены уравнения для производящего функционала систем, обладающих внутренней симметрией и связями. В рамках гармонического приближения определены статистическая сумма и моменты параметра порядка в зависимости от параметра неаддитивности. Развита теория возмущений, использование которой позволяет найти поправки произвольного порядка к указанным величинам.

Ключевые слова: параметр неаддитивности, производящий функционал, статистическая сумма.

DOI: $10.4213 / \operatorname{tmf} 8408$

\section{1. ВВЕДЕНИЕ}

Статистическая физика основывается, как известно, на предположении о перемешивании фазового пространства [1], [2]. Согласно этому предположению в ходе эволюции выделенный объем быстро сжимается по одним направлениям и расширяется по другим, приобретая с течением времени настолько разветвленную форму, что его точки можно найти в любой конечной части фазового пространства. Будучи дополненной предположением о бесконечном числе степеней свободы, гипотеза перемешивания приводит к распределению Гиббса, из которого следует аддитивность термодинамических потенциалов. При этом выполняются следующие условия [3]:

* Сумский государственный университет, Сумы, Украина

${ }^{\dagger}$ Институт прикладной физики НАН Украины, Сумы, Украина. E-mail: yushchenko@phe.sumdu.edu.ua 
- кинетическое - перемешивание протекает экспоненциально быстро (это обеспечивает хорошо развитую хаотическую структуру и требует положительности наибольшего из показателей Ляпунова);

- динамическое - все силы, включая те, что обеспечивают микроскопическую память, являются короткодействующими (в результате стохастический процесс имеет марковский характер);

- геометрическое - фазовое пространство обладает обычными свойствами непрерывности, гладкости, евклидовости и т. п.

В последнее время обнаружено множество систем, проявляющих неаддитивное поведение. К ним относятся ферромагнетики, спиновые стекла, двумерная электронная плазма в турбулентном режиме, системы с аномальной диффузией Леви, гранулированные системы, твердые тела, подвергнутые ионной бомбардировке, гравитационные системы, солнечные нейтрино, черные дыры, элементарные частицы, сталкивающиеся с высокой энергией, квантовые системы, проявляющие эффекты запутывания, и многие другие [3]. В таких системах экспоненциально быстрое перемешивание приобретает степенной характер, в результате чего происходит только слабая хаотизация фазового пространства. Кроме того, этим системам присущи эффекты дальнодействия, немарковское поведение, мультифрактальные граничные или патологические начальные условия, некоторые специальные механизмы диссипации и т. д.

C формальной точки зрения теория неаддитивных систем основывается на деформации логарифмической и экспоненциальной функций, которая модифицирует энтропию Больцмана-Гиббса таким образом, что функция распределения приобретает либо дальнодействующие степеннь́е асимптотики [4]-[11], либо обрезается на конечных значениях энергии [12], [13]. Характерной особенностью неаддитивных систем является самоподобие их фазового пространства, объем которого остается неизменным при деформации, комбинирующей сжатие (растяжение) координаты и растяжение (сжатие) импульса [14].

С другой стороны, деформация перестановочных соотношений в квантовой теории позволила развить нетривиальные физические представления таких объектов, как черные дыры и анионные сверхпроводники [15]. Эти представления основаны на формализме квантовых групп, который сводится к так называемому $q$-исчислению [16]-[18], впервые введенному Хейне и Джексоном [19], [20] при изучении базоводеформированных гипергеометрических рядов [21], [22]. С математической точки зрения $q$-исчисление представляет собой наиболее удобный формализм для описания мультифрактальных множеств, которые образуются в результате многократного действия оператора дилатации, определяющего производную Джексона [14]. Более того, оказывается [6], [23], что для q-деформированных бозонов и фермионов естественное обобщение термостатистики основано на $q$-исчислении, а стационарное решение деформированного уравнения Фоккера-Планка представляет собой $q$-аналог экспоненциальной функции в представлении базово-деформированных гипергеометрических рядов [12], [13]. Такого рода системы обнаруживают дискретную масштабную инвариантность [24], описание которой [25] достигается использованием производной и интеграла Джексона (например, свободная энергия спиновых систем, определенных на иерархической решетке, сводится к однородной функции, представляющей $q$-интеграл [26]).

Следует отметить еще один пример приложения статистической теории неаддитивных систем - к описанию объектов конечного размера, важность исследования 
которых возросла с развитием нанотехнологий. Действительно, при конечном числе частиц $N$ параметр неаддитивности принимает значение [27]

$$
q=\left(1-\frac{\alpha}{d} N^{-1}\right)^{-1}
$$

где $\alpha$ - показатель подобия координатной зависимости гамильтониана (например, для гармонического осциллятора $\alpha=2), d$ - размерность системы. Короткодействующие потенциалы $(\alpha>0)$ характеризуются значениями $q \geqslant 1$, а дальнодействующим $(-d \leqslant \alpha \leqslant 0$ ) отвечает величина $q \leqslant 1$ (при $\alpha<-d$ применима статистика Больцмана-Гиббса [28]). В термодинамическом пределе $N \rightarrow \infty$ получаем значение $q=1$, отвечающее обычной статистике, а со спаданием числа частиц $N$ разность $|q-1|$ возрастает, достигая максимальной величины $\alpha /(d-\alpha)$ при $\alpha>0$ и $|\alpha| /(d+|\alpha|)$ при $\alpha<0$.

Настоящая работа посвящена рассмотрению неаддитивных статистических систем в рамках полевого формализма, который, основываясь на использовании методов квантовой теории поля, представляет собой один из наиболее мощных инструментов исследования [29], [30]. Формальной основой стандартной полевой схемы является, как известно, производящий функционал, который представляет собой обобщенное преобразование Фурье-Лапласа, дающее переход от распределения флуктуирующего параметра порядка (амплитуды гидродинамической моды) к вспомогательному полю [31]. Благодаря экспоненциальному характеру этого преобразования определение корреляторов параметра порядка достигается дифференцированием производящего функционала по указанному полю.

Представленная схема становится несостоятельной при переходе к неаддитивным статистическим системам, поскольку экспонента Больцмана-Гиббса принимает биномиальную форму Цаллиса [3]. В результате построение генерирующего функционала требует деформации преобразования Фурье-Лапласа, а вместо обычного дифференцирования следует использовать оператор, относительно которого инвариантно ядро деформированного преобразования [32]. Так, например, при построении статистической теории самоподобно распределенных полей роль базисной функции играет степенна́я зависимость, для которой производящий функционал сводится к пребразованию Меллина статистического функционала, а оператор дифференцирования представляет собой производную Джексона [2], [33].

Работа построена следующим образом. В разделе 2 приводятся основные соотношения теории Мартина-Сиггиа-Роуза [34], использование которой позволяет определить эффективный лагранжиан системы исходя из стохастического уравнения движения и найти пару уравнений для сопряженных координаты и импульса, роль которых играют наиболее вероятные значения амплитуд гидродинамической моды и ее флуктуаций. Раздел 3 посвящен обобщению полевой схемы для неаддитивных систем. Показано, что деформация статистического распределения не изменяет уравнения эволюции наиболее вероятных значений параметра порядка и амплитуды его флуктуаций, тогда как вероятность реализации различных фазовых траекторий существенно зависит от параметра неаддитивности. В разделе 4 найден производящий функционал неаддитивной системы и установлена его связь с корреляторами; введена пара аддитивных производящих функционалов, разложение которых дает набор многоточечных функций Грина и их собственно-энергетических частей; найдены уравнения для производящего функционала систем, обладающих 
внутренней симметрией и связями. Центральное место занимает раздел 5, посвященный описанию термодинамических свойств неаддитивной системы: сначала используется простейшее гармоническое приближение, в рамках которого определены явные выражения для одноузельной статистической суммы и моментов параметра порядка в зависимости от параметра неаддитивности; затем развита теория возмущений, использование которой позволяет найти поправки произвольного порядка к указанным величинам. В разделе 6 содержится обсуждение полученных результатов. И наконец, приложение содержит основные сведения из $q$-деформированной алгебры [35], лежащей в основе неаддитивной статистики.

\section{2. ОСНОВНЫЕ СООТНОШЕНИЯ ТЕОРЕТИКО-ПОЛЕВОЙ СХЕМЫ}

Представим поведение стохастической системы пространственно-временно́й зависимостью $x(\mathbf{r}, t)$ амплитуды гидродинамической моды, среднее значение которой сводится к параметру порядка. Для описания этой зависимости будем исходить из уравнения Ланжевена [36]

$$
\dot{x}(\mathbf{r}, t)-D \nabla^{2} x=-\gamma \frac{\partial F}{\partial x}+\zeta(\mathbf{r}, t) .
$$

Здесь точка означает временну́ю производную, $\nabla \equiv \partial / \partial \mathbf{r}, D$ - параметр неоднородности, $\gamma$ - кинетический коэффициент, $F(x)$ - свободная энергия, $\zeta(\mathbf{r}, t)$ - стохастическая добавка, определенная условиями белого шума:

$$
\langle\zeta(\mathbf{r}, t)\rangle=0, \quad\langle\zeta(\mathbf{r}, t) \zeta(\mathbf{0}, 0)\rangle=\gamma T \delta(\mathbf{r}) \delta(t)
$$

где угловые скобки означают усреднение по гауссову распределению величины $\zeta, T-$ температура, задающая интенсивность шума. Далее удобно ввести единицы измерения $(\gamma T)^{2} / D^{3}, \gamma T / D, D^{3} / \gamma^{3} T^{2}, D^{3} /(\gamma T)^{2}$ для времени $t$, координаты $\mathbf{r}$, плотности термодинамического потенциала $F$ и стохастической переменной $\zeta$ соответственно (при этом подразумевается, что амплитуда гидродинамической моды $x$ изначально принята безразмерной). В результате уравнение движения (2) принимает канонический вид

$$
\dot{x}(\mathbf{r}, t)=-\frac{\delta \mathcal{F}}{\delta x}+\zeta(\mathbf{r}, t),
$$

где использована краткая форма записи вариационной производной:

$$
\frac{\delta \mathcal{F}}{\delta x} \equiv \frac{\delta \mathcal{F}\{x(\mathbf{r}, t)\}}{\delta x(\mathbf{r}, t)}=\frac{\partial F(x)}{\partial x}-\nabla^{2} x, \quad \mathcal{F}\{x\} \equiv \int\left[F(x)+\frac{1}{2}(\nabla x)^{2}\right] d \mathbf{r} .
$$

Кроме того, в формуле (3) пропадает коэффициент $\gamma T$, а распределение переменной $\zeta$ приобретает стандартную гауссову форму

$$
P_{0}\{\zeta\}=\frac{1}{\sqrt{2 \pi}} e^{(-1 / 2) \int \zeta^{2}(\mathbf{r}, t) d \mathbf{r} d t} .
$$

Статистическая теория поля основывается на методе производящего функционала [31], [34]:

$$
\mathcal{Z}\{u(\mathbf{r}, t)\}=\int Z\{x\} e^{\int u x d \mathbf{r} d t}\{\delta x\}, \quad\{\delta x\} \equiv \prod_{\mathbf{r}, t} \delta x(\mathbf{r}, t),
$$


который представляет собой функциональное преобразование Лапласа обобщенной статистической суммы

$$
\mathcal{Z}\{x(\mathbf{r}, t)\} \equiv\left\langle\prod_{(\mathbf{r}, t)} \delta\left\{\dot{x}+\frac{\delta \mathcal{F}}{\delta x}-\zeta\right\} \operatorname{det}\left|\frac{\delta \zeta}{\delta x}\right|\right\rangle .
$$

В ее определении $\delta$-функционал учитывает уравнение Ланжевена (4), а детерминант определяет переход от континуального интегрирования по $\zeta$ к $x$.

Варьирование производящего функционала (7) по пробному полю $u(\mathbf{r}, t)$ позволяет найти корреляторы наблюдаемой величины $x(\mathbf{r}, t)$ :

$$
\left\langle x\left(\mathbf{r}_{1}, t_{1}\right) \ldots x\left(\mathbf{r}_{n}, t_{n}\right)\right\rangle=\left.\frac{1}{\mathcal{Z}\{u=0\}}\left[\mathcal{D}_{u\left(\mathbf{r}_{1}, t_{1}\right)} \ldots \mathcal{D}_{u\left(\mathbf{r}_{n}, t_{n}\right)}\right] \mathcal{Z}\{u(\mathbf{r}, t)\}\right|_{u(\mathbf{r}, t)=0},
$$

где $\mathcal{Z}\{0\} \equiv \mathcal{Z}\{u(\mathbf{r}, t)=0\}, \mathcal{D}_{u(\mathbf{r}, t)} \equiv \delta / \delta u(\mathbf{r}, t)$. С учетом тождественности $N$ частиц, составляющих систему, статистическая сумма определяется выражением

$$
Z_{N}=\frac{1}{N !} \mathcal{Z}\{0\}=\frac{1}{N !} \int \mathcal{Z}\{x(\mathbf{r}, t)\}\{\delta x\} .
$$

Кроме корреляторов наблюдаемых величин, использование производящего функционала (7) позволяет проследить за эволюцией наиболее вероятных значений стохастической переменной $x$ и ее дисперсии. С этой целью представим $\delta$-функционал в выражении (8) функциональным разложением Лапласа

$$
\delta\{x(\mathbf{r}, t)\}=\int_{-i \infty}^{i \infty} e^{-\int p x d \mathbf{r} d t}\{\delta p\}
$$

по полю духов $p(\mathbf{r}, t)$, физический смысл которого будет установлен ниже. Тогда после усреднения по распределению (6) статистический функционал (8) принимает стандартный вид

$$
\mathcal{Z}\{x(\mathbf{r}, t)\}=\int e^{-S\{x(\mathbf{r}, t), p(\mathbf{r}, t)\}}\{\delta p\},
$$

где эффективное действие $S=\int \mathcal{L} d t$ определяется лагранжианом

$$
\mathcal{L}=p\left(\dot{x}+\frac{\delta \mathcal{F}}{\delta x}\right)-\frac{p^{2}}{2}, \quad \frac{\delta \mathcal{F}}{\delta x} \equiv-f-\nabla^{2} x, \quad f \equiv-\frac{\partial F}{\partial x} .
$$

Далее следует использовать уравнение Эйлера

$$
\frac{\partial \mathcal{L}}{\partial \mathbf{x}}-\frac{d}{d t} \frac{\partial \mathcal{L}}{\partial \dot{\mathbf{x}}}-\nabla \frac{\partial \mathcal{L}}{\partial \nabla \mathbf{x}}+\nabla^{2} \frac{\partial \mathcal{L}}{\partial \nabla^{2} \mathbf{x}}=\frac{\partial \mathcal{R}}{\partial \dot{\mathbf{x}}}, \quad \mathbf{x} \equiv\{x, p\}
$$

с диссипативной функцией $\mathcal{R}=\dot{x}^{2} / 2$ [37]. В результате получаем уравнения для наиболее вероятных значений статистических полей $x(\mathbf{r}, t), p(\mathbf{r}, t)$ :

$$
\begin{gathered}
\dot{x}-\nabla^{2} x=f+p, \\
\dot{p}+\dot{x}+\nabla^{2} p=-f^{\prime} p,
\end{gathered}
$$

где штрих означает дифференцирование по $x$. Сравнение уравнения (15), записанного в виде $\dot{x}=-\delta \mathcal{F} / \delta x+p$, с выражением (4) показывает, что поле $p(\mathbf{r}, t)$ представляет наиболее вероятные значения амплитуды флуктуаций $\zeta(\mathbf{r}, t)$ сопряженной силы $f$. 


\section{3. ЭВОЛЮЦИЯ НЕАДДИТИВНОЙ СИСТЕМЫ}

Сравнение деформированной $\delta$-функции (см. формулу (П.10) в приложении) со стандартным функциональным представлением (11) показывает, что с точностью до замены числа $k$ на комплексное поле $i p(\mathbf{r}, t)$ эти выражения имеют одинаковую форму. Благодаря этому статистический функционал

$$
\mathcal{Z}_{q}\{x(\mathbf{r}, t)\}=\frac{2 \pi}{2-q} \int e_{q}^{-S_{q}\{x(\mathbf{r}, t), p(\mathbf{r}, t)\}}\{\delta p\}
$$

отличается от стандартного представления (12) несущественным множителем и заменой обычной экспоненты на деформированную (П.2) (см. приложение). В результате деформация статистической системы не влияет на форму лагранжиана (13). Поэтому варьирование функционала (17) приводит к тем же уравнениям эволюции (15), (16), что и для аддитивной статистической системы.

Таким образом, деформация фазового пространства не изменяет форму траекторий, по которым протекает эволюция неаддитивной системы. Определим плотность свободной энергии разложением Ландау

$$
F=\frac{\varepsilon}{2} x^{2}+\frac{1}{4} x^{4}
$$

с безразмерной температурой $\varepsilon$, отсчитанной от точки превращения; кроме того, градиентные слагаемые $\nabla^{2} x$ и $\nabla^{2} p$ аппроксимируем линейными функциями $x / \xi^{2}$, $p / \lambda^{2}$ с масштабами $\xi$ и $\lambda$. В результате система $(15),(16)$ принимает вид

$$
\begin{aligned}
& \dot{x}=-\left[\left(\varepsilon-\xi^{-2}\right)+x^{2}\right] x+p, \\
& \dot{p}+\dot{x}=\left[\left(\varepsilon-\lambda^{-2}\right)+3 x^{2}\right] p .
\end{aligned}
$$

Поскольку уравнения (19), (20) симметричны относительно одновременного обращения знаков переменных $x, p$, то отвечающие им фазовые портреты (рис. 1) имеют центрально-симметричную форму.

Из рис. 1а видно, что при сильной неоднородности флуктуаций выше точки перехода, когда выполняются условия $0<\varepsilon<\lambda^{-2}$, реализуются устойчивый узел $O$, расположенный в начале координат $x_{0}=0, p_{0}=0$, и пара центрально-симметричных седел $S_{1,2}$. Очевидно, такая картина отвечает однородному неупорядоченному состоянию. С ослаблением неоднородности в распределении флуктуаций до такой степени, что нарушается неравенство $\varepsilon<\lambda^{-2}$, и усилением неоднородности распределения параметра порядка, обеспечивающим условие $\varepsilon<\xi^{-2}$ (рис. 1б), седла $S_{ \pm}$симметрично смещаются на ось абсцисс, а узел $O$ трансформируется в фокус. Уместно предположить, что такая ситуация представляет эволюцию неупорядоченной системы через стадию гетерофазных флуктуаций. И, наконец, с размытием пространственных распределений амплитуды флуктуаций и параметра порядка, когда одновременно выполняются неравенства $\varepsilon>\lambda^{-2}, \varepsilon>\xi^{-2}$, происходит обратная бифуркация пары седел $S_{ \pm}$и узла $O$ в седло $S$, расположенное в начале координат. Согласно рис. 1в такой случай не отвечает какому-либо стационарному состоянию.

Переход в упорядоченную фазу $\varepsilon<0$ (рис. 1г-1е) обеспечивает выполнение условий $\varepsilon<\lambda^{-2}, \varepsilon<\xi^{-2}$, при которых возможные конфигурации фазовых портретов определяются наличием пяти особых точек - седла $S$, расположенного в начале координат, центрально-симметричных устойчивых узлов/фокусов $N_{1,2} / F_{1,2}$ и пары седел $S_{ \pm}$, симметрично расположенных на оси абсцисс. Согласно рис. 1г размытие 

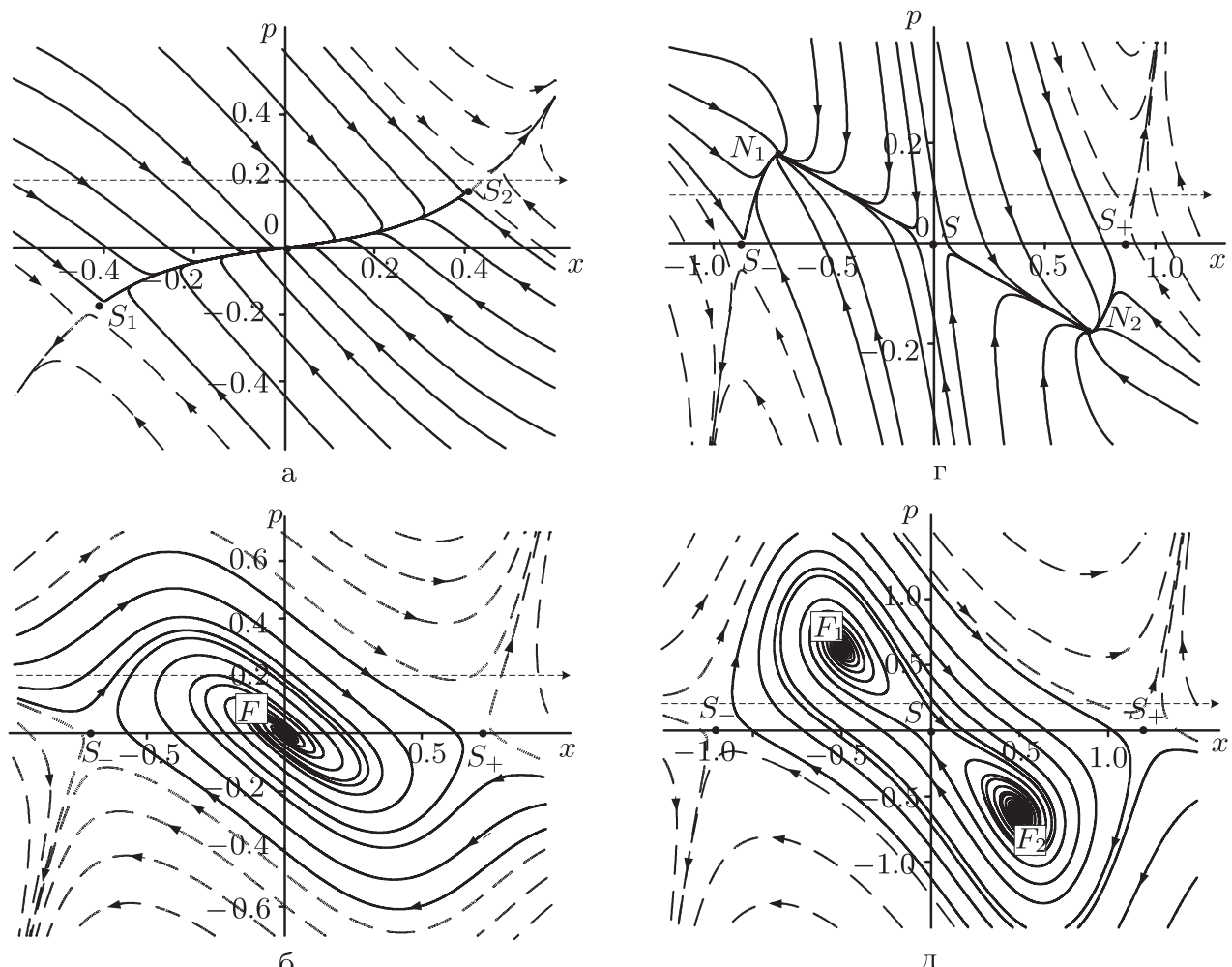

б

д

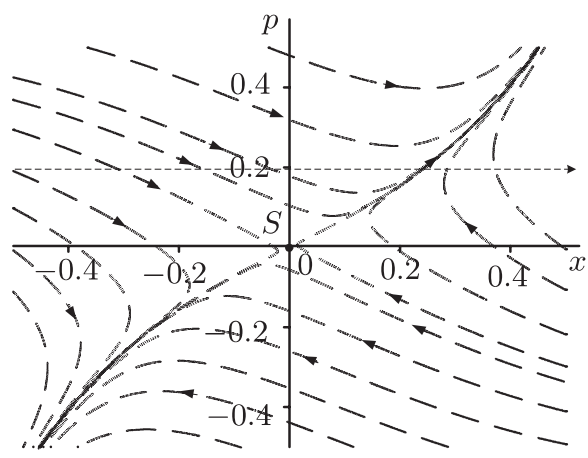

B

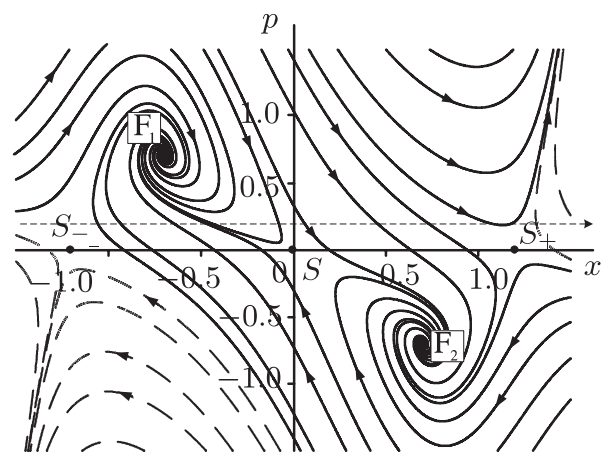

e

Рис. 1. Фазовые портреты: выше точки перехода ( $\varepsilon=0.5)$ при $\xi=2, \lambda=1$ (a), $\xi=1, \lambda=2$ (б), $\xi=10, \lambda=10$ (в) и ниже точки перехода $(\varepsilon=-0.5)$ при $\xi=2, \lambda=1$ (г), $\xi=1, \lambda=2$ (д), $\xi=1, \lambda=1$ (е). Штриховами линиями указаны траектории, реализуемые с малой вероятностью, пунктирные лучи отвечают постоянной амплитуде флуктуаций $p=0.2$.

неоднородности параметра порядка за счет роста масштаба $\xi$ приводит к ослаблению колебательного режима вблизи стационарных состояний $N_{1,2} / F_{1,2}$, а размытие неоднородности распределения флуктуаций за счет увеличения масштаба $\lambda$ (рис. 1д) усиливает его. В явном виде влияние масштабов $\xi, \lambda$ определяется показателями Ля- 
пунова

$$
\begin{gathered}
\lambda_{1,2}=-(\alpha+\beta) \pm \sqrt{\beta^{2}-\alpha-6 x_{0} p_{0}}, \\
\alpha \equiv\left(\varepsilon-\xi^{-2}\right)+3 x_{0}^{2}, \quad \beta \equiv \frac{1}{2}\left(1+\xi^{-2}+\lambda^{-2}\right),
\end{gathered}
$$

где $x_{0}, p_{0}$ - координаты особых точек.

Как уже указывалось, деформация неаддитивной статистической системы не изменяет вид фазовых портретов. Покажем, что она существенно сказывается на вероятности реализации фазовых траекторий

$$
P_{q}\{p(x)\}=Z_{q}^{-1} e_{q}^{-S\{p(x)\}} .
$$

Здесь эффективное действие $S\{p(x)\}=S_{\min }\{x(\mathbf{r}, t), p(\mathbf{r}, t)\}$ принимает минимальные значения, отвечающие оптимальным фазовым траекториям эволюции системы, а статистическая сумма определяется условием нормировки

$$
Z_{q}=\int e_{q}^{-S\{p(x)\}}\{\delta p\}, \quad\{\delta p\} \equiv \prod_{x} \delta p(x) .
$$

Для представления функциональной зависимости (22) в графическом виде будем проводить перебор фазовых траекторий вдоль лучей, показанных на рис. 1 при постоянной амплитуде флуктуаций $p=0.2$. В результате получаем распределения вероятностей реализации этих траекторий, показанные на рис. 2. Из них видно, что указанные вероятности определяются положениями сепаратрис на фазовых портретах (на рис. 1 траектории, отвечающие конечным вероятностям, выделены сплошными линиями). Обращает на себя внимание тот факт, что в однородном неупорядоченном состоянии (рис. 1a) с конечной вероятностью реализуются траектории, ограниченные только большими значениями параметра порядка. С другой стороны, гетерофазные флуктуации неупорядоченного состояния (рис. 1б) и изменения параметра порядка во всех режимах упорядоченного состояния (рис. $1 \Gamma-1 \mathrm{e})$ ограничены как сверху, так и снизу. Сравнение рис. 1г с рис. 1д, 1е показывает, что трансформация притягивающих узлов в фокусы приводит к более рельефному изменению вероятности реализации различных траекторий. С другой стороны, из рис. 3 видно, что при значительном росте параметра неаддитивности все разрешенные траектории становятся практически равновероятными.

\section{4. ПРОИЗВОДЯЩИЕ ФУНКЦИОНАЛЫ}

Рассмотрим неаддитивный статистический ансамбль, состоящий из $N$ частиц, распределенных по состояниям $\mathbf{q}=\left\{\mathbf{r}_{i}, \mathbf{p}_{i}\right\}$ в фазовом пространстве с координатами $\mathbf{r}_{i}$ и импульсами $\mathbf{p}_{i}$, от которых зависит гамильтониан $H=H(\mathbf{q})$. При пространственно-временной зависимости параметра порядка $x=x(\mathbf{r}, t)$ статистический функционал

$$
\mathcal{Z}_{q}\{x\}=\int e_{q}^{-\beta H(\mathbf{q})} \delta[x-x(\mathbf{q})] d \mathbf{q},
$$

зависящий от обратной температуры $\beta \equiv T^{-1}$, связан с эффективным действием $S_{q}\{x\}$ определением

$$
\mathcal{Z}_{q}\{x\}:=e_{q}^{-S_{q}\{x\}} .
$$

В согласии с деформированным преобразованием Фурье (П.8) производящий функционал представляется обобщенным преобразованием Лапласа:

$$
\mathcal{Z}_{q}\{u(\mathbf{r}, t)\}:=\int \mathcal{Z}_{q}\{x\} \otimes_{q} e_{q}^{u \cdot x}\{\delta x\}=\int e_{q}^{u \cdot x-S_{q}\{x\}}\{\delta x\},
$$



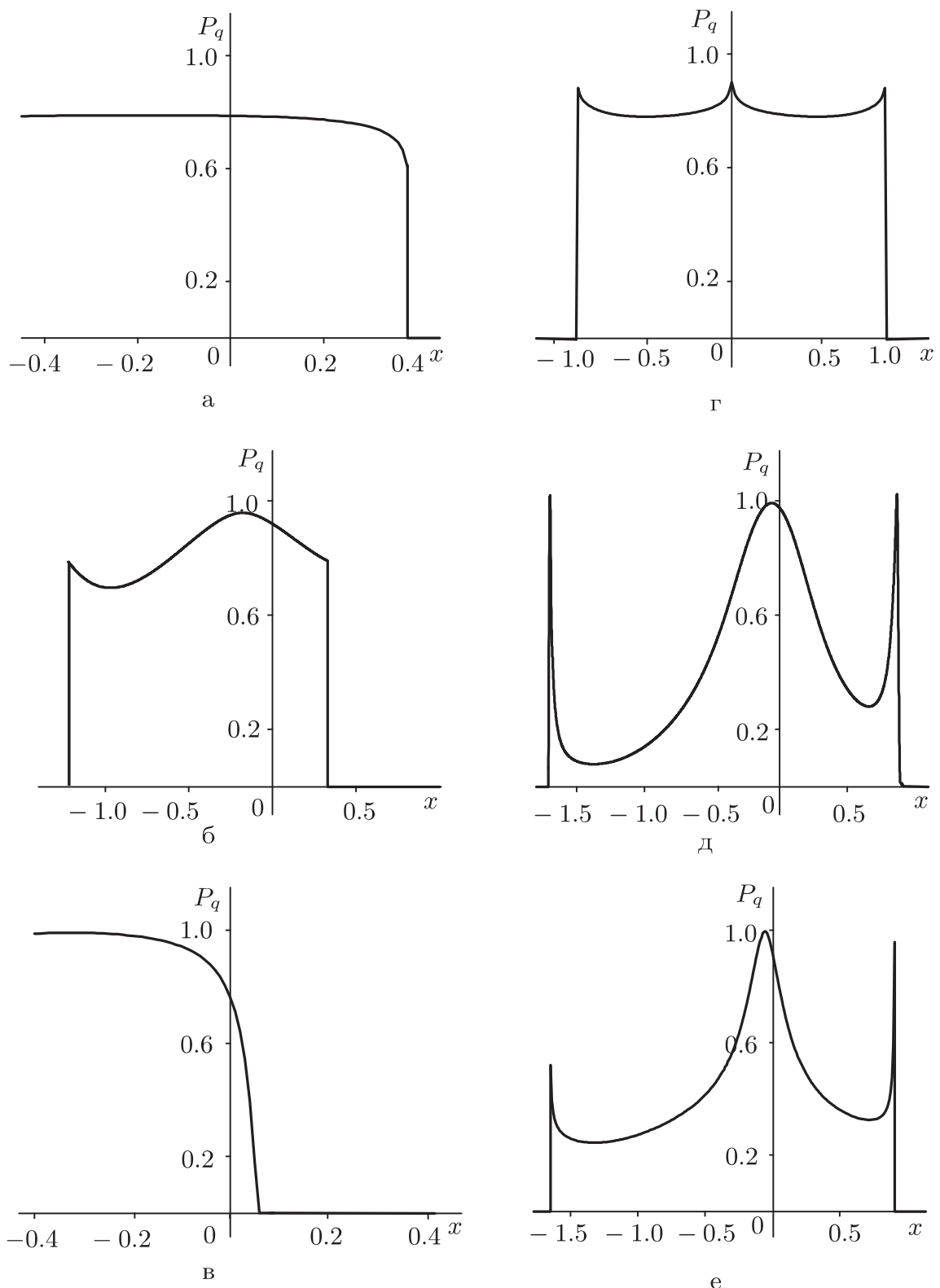

Рис. 2. Распределения вероятностей реализации различных траекторий, отвечающие изменениям параметра порядка $x$ вдоль лучей, соответствующих постоянной амплитуде флуктуаций $p=0.2$ при параметре неаддитивности $q=0.4$.

где обозначено $u \cdot x \equiv \int u x d \mathbf{r} d t$. Поскольку экспонента Цаллиса $e_{q}^{x}$ сохраняет свою форму под действием оператора $[1+(1-q) x]_{+} d / d x$, то далее удобно ввести $q$-вариационную производную $\mathcal{D}_{x(\mathbf{r}, t)}^{q}$, действие которой на произвольный функцио- 


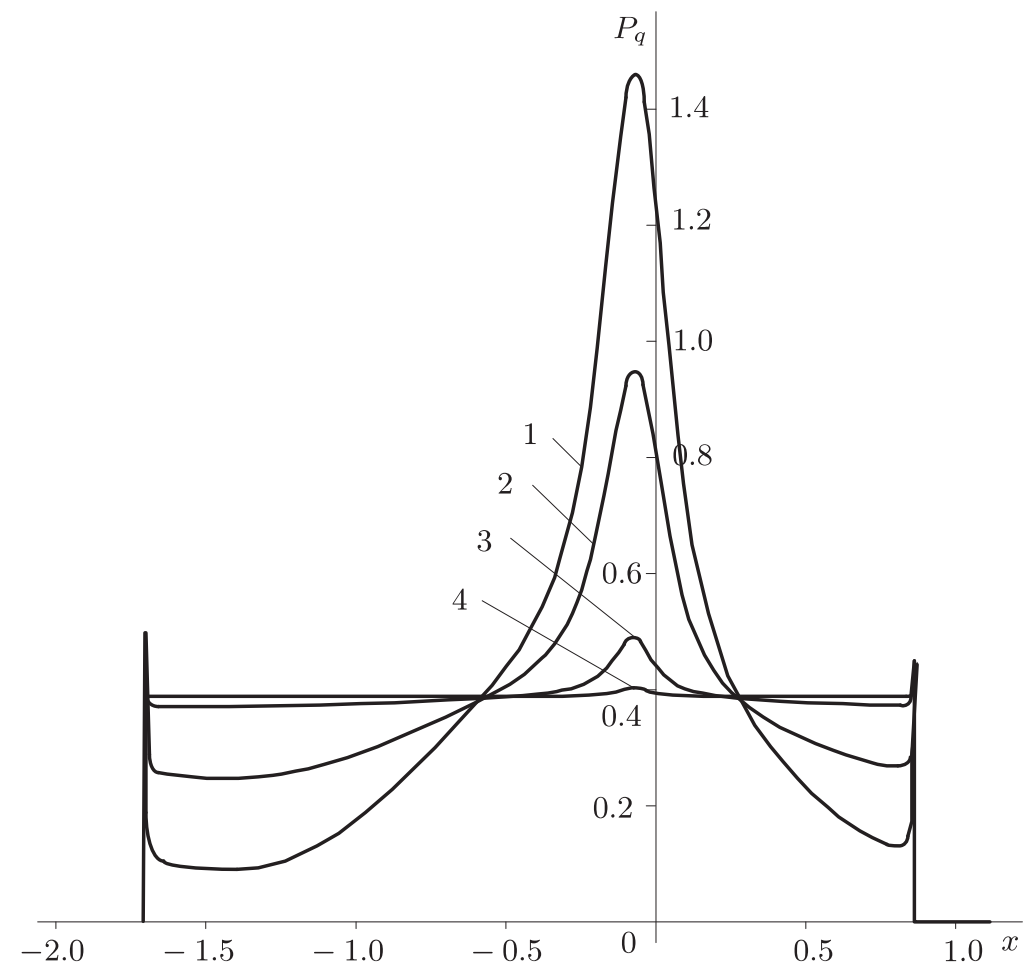

Рис. 3. Распределения вероятностей реализации фазовых траекторий на рис. 1д при параметрах неаддитивности $q=0.4,1.0,10,100$ (кривые $1-4$ соответственно).

нал $f=f\{x(\mathbf{r}, t)\}$ задается равенством

$$
\mathcal{D}_{x(\mathbf{r}, t)}^{q} f:=\left[e_{q}^{f}\right]^{1-q} \frac{\delta f}{\delta x(\mathbf{r}, t)} .
$$

Тогда $n$-кратное варьирование производящего функционала (26) дает $n$-точечный коррелятор

$$
\begin{aligned}
\left\langle x\left(\mathbf{r}_{1}, t_{1}\right)\right. & \left.\ldots x\left(\mathbf{r}_{n}, t_{n}\right)\right\rangle=\frac{1}{\left.\mathcal{Z}_{q}\{u\}\right|_{u=0}} \times \\
& \times\left.\left[\mathcal{D}_{u\left(\mathbf{r}_{1}, t_{1}\right)}^{q} \ldots \mathcal{D}_{u\left(\mathbf{r}_{n}, t_{n}\right)}^{q}\right] \mathcal{Z}_{q}\{u(\mathbf{r}, t)\}\right|_{u\left(\mathbf{r}_{1}, t_{1}\right), \ldots, u\left(\mathbf{r}_{n}, t_{n}\right)=0},
\end{aligned}
$$

имеющий ту же структуру, что и в стандартной теории поля [31]. С учетом тождественности $N$ частиц, составляющих систему, ее статистическая сумма определяется выражениями

$$
Z_{N q}:=\frac{1}{N !} \mathcal{Z}_{q}\{u=0\}=\frac{1}{N !} \int e_{q}^{-S_{q}\{x\}}\{\delta x\} .
$$

Как и в обычной полевой схеме [31], неудобство производящего функционала (26) состоит в его неаддитивности [38]. Для устранения этого недостатка введем функционал

$$
\mathcal{G}_{q}\{u\}:=\ln _{q}\left(\mathcal{Z}_{q}\{u\}\right),
$$


представляющий собой деформированный логарифм (П.3) (см. приложение) выражения (26). Если в функциональной зависимости (30) удобно перейти от вспомогательного поля $u(\mathbf{r}, t)$ к параметру порядка $x(\mathbf{r}, t)$, то следует провести преобразование Лежандра

$$
\Gamma_{q}\{x\}:=u \cdot x-\mathcal{G}_{q}\{u\},
$$

приводящее к сопряженному функционалу $\Gamma_{q}=\Gamma_{q}\{x\}$. Пара функционалов $\mathcal{G}_{q}\{u\}$, $\Gamma_{q}\{x\}$ играет роль сопряженных потенциалов, вариация которых дает уравнения состояний

$$
x(\mathbf{r}, t)=\mathcal{D}_{u}^{q} \mathcal{G}_{q}\{u\} \quad \Leftrightarrow \quad u(\mathbf{r}, t)=\mathcal{D}_{x}^{q} \Gamma_{q}\{x\} .
$$

Первое из них представляет собой обобщение термодинамического определения параметра порядка, второе следует из преобразования Лежандра (31) после его вариации по параметру порядка. Являясь аналитическими функционалами, потенциалы $\mathcal{G}_{q}\{u\}, \Gamma_{q}\{x\}$ представляются следующими рядами:

$$
\begin{gathered}
\mathcal{G}_{q}\{u\}=\sum_{n=1}^{\infty} \frac{1}{[n]_{q} !} \sum_{1, \ldots, n} \mathcal{G}_{1, \ldots, n}^{(n)} u_{1} \ldots u_{n} \\
\Gamma_{q}\{x\}=\sum_{n=1}^{\infty} \frac{1}{[n]_{q} !} \sum_{1, \ldots, n} \Gamma_{i, \ldots, n}^{(n)} \eta_{1} \ldots \eta_{n}, \quad \eta_{i} \equiv x_{i}-\mathcal{G}_{i}^{(1)} .
\end{gathered}
$$

Здесь $[n]_{q}$ ! - факториалы $q$-деформированных чисел $[n]_{q}=[1+(1-q)(n-1)]_{+}^{-1} n=$ $\left(e_{q}^{n}\right)^{-(1-q)} n$, индексы $i=1, \ldots, n$ означают координаты $\mathbf{r}_{i}, t_{i}$ пространства-времени, ядра $\mathcal{G}_{1, \ldots, n}^{(n)}, \Gamma_{1, \ldots, n}^{(n)}$ сводятся к $n$-частичным функциям Грина и их неприводимым частям соответственно.

Подобно стандартной полевой схеме [31] производящий функционал (26) удовлетворяет некоторым формальным соотношениям. Первое из них отображает симметрию системы по отношению к вариации $\delta x_{i}=\epsilon f_{i}\{x\}$, заданной аналитическим функционалом $f_{i}=f_{i}\{x\}$ в пределе $\epsilon \rightarrow 0$. При варьировании последнее подынтегральное выражение в функционале (26) преобразуется следующим образом:

$$
\begin{aligned}
& e_{q}^{-S\{x+\delta x\}+u(x+\delta x)} \simeq \\
& \quad \simeq\left[(1+(1-q)(-S\{x\}+u \cdot x))+(1-q)\left(-\frac{\partial S}{\partial x_{i}}+u_{i}\right) \delta x_{i}\right]_{+}^{1 /(1-q)}= \\
& \quad=e_{q}^{-S\{x\}+u \cdot x}\left[1+\frac{(1-q)\left(-\partial S / \partial x_{i}+u_{i}\right)}{1+(1-q)(-S\{x\}+u \cdot x)} \delta x_{i}\right]_{+}^{1 /(1-q)} \simeq \\
& \quad \simeq e_{q}^{-S\{x\}+u \cdot x}\left[1+\left(-\frac{\partial S}{\partial x_{i}}+u_{i}\right) \delta_{q} x_{i}\right]_{+}
\end{aligned}
$$

где подразумевается суммирование по повторяющимся индексам и введен $q$-вириал (cp. с (27))

$$
\delta_{q} x_{i}:=\left[e_{q}^{-S\{x\}+u \cdot x}\right]^{q-1} \delta x_{i} .
$$

С другой стороны, якобиан перехода от $x$ к $x+\delta_{q} x$ дает множитель $1+\left(\partial f_{i} / \partial_{q} x_{i}\right) \epsilon$, определенный оператором q-дифференцирования $\left(\partial f / \partial_{q} x\right)=\left(e_{q}^{f}\right)^{1-q}(\partial f / \partial x)$ типа (27). Собирая слагаемые, пропорциональные бесконечно малой величине $\epsilon$, из свойства инвариантности производящего функционала (26) получаем

$$
\left[f_{i}\left\{\mathcal{D}_{u}^{q}\right\}\left(\frac{\partial S}{\partial x_{i}}\left\{\mathcal{D}_{u}^{q}\right\}-u_{i}\right)-\frac{\partial f_{i}}{\partial x_{i}}\left\{\mathcal{D}_{u}^{q}\right\}\right] \mathcal{Z}_{q}\{u\}=0 .
$$


Здесь использовано первое уравнение состояний (32) для операторного представления

$$
f_{i}\{x\} e_{q}^{-S\{x\}+u \cdot x}=f_{i}\left\{\mathcal{D}_{u}^{q}\right\} e_{q}^{-S\{x\}+u \cdot x} .
$$

При $f_{i}\{x\}=$ const выражение (37) принимает упрощенный вид, следующий непосредственно из производящего функционала (26) после его варьирования по полю $x$.

Второе из упомянутых соотношений позволяет учесть наличие произвольных связей $F_{j}\{x\}=0, j=1,2, \ldots$, для искомого набора значений поля $x=x(\mathbf{r}, t)$. Учет этих связей достигается подстановкой $\delta$-функционала $\delta_{q}\{F\}$ в подынтегральное выражение (26), что приводит к удлиненному производящему функционалу

$$
\mathcal{Z}_{q}^{(F)}\{u, v\}:=\int e_{q}^{-S\{x\}+u \cdot x+v \cdot F}\{\delta x\}\{\delta v\} .
$$

Его варьирование по вспомогательному полю $v=v\{\mathbf{r}, t\}$ дает искомый результат

$$
F_{i}\left\{\mathcal{D}_{v}^{q}\right\} \mathcal{Z}_{q}^{(F)}\{u, v\}=0 .
$$

В сравнении со стандартной полевой схемой [31] главная особенность выражений $(37),(39)$ состоит в том, что они содержат $q$-вариационную производную (27).

\section{5. ТЕРМОДИНАМИКА НЕАДДИТИВНОЙ СИСТЕМЫ}

Рассмотрим сначала гармоническое приближение, в рамках которого эффективное действие $S_{q}^{(0)}\{x\}=\int \mathcal{L}_{q}^{(0)}(x(t)) d t$ определяется суммой

$$
\mathcal{L}_{q}^{(0)}=\sum_{i=1}^{N} \lambda_{q}^{(0)}\left(x_{i}\right), \quad \lambda_{q}^{(0)}(x)=\frac{x^{2}}{[2]_{q} \Delta^{2}}, \quad[2]_{q} \equiv \frac{2}{|2-q|} .
$$

Здесь каждое слагаемое задается обратной кривизной $\Delta^{2}$ и параметром неаддитивности $q$ (ср. с работой [38]). С учетом правил (П.4), (П.5) производящий функционал (26) представляется деформированным произведением

$$
\mathcal{Z}_{q}^{(0)}\{u\}=z_{q}^{(0)}\left(u_{1}\right) \otimes_{q} \cdots \otimes_{q} z_{q}^{(0)}\left(u_{N}\right)
$$

одночастичных составляющих

$$
z_{q}^{(0)}(u)=\int \exp _{q}\left[\int\left(u x-\frac{x^{2}}{[2]_{q} \Delta^{2}}\right) d \mathbf{r} d t\right] \delta x .
$$

При нахождении статистической суммы $Z_{q N}^{(0)}$ следует учесть тождественность частиц, число перестановок которых равно $q$-факториалу (П.6) при $n=N$. В результате приходим к определению

$$
Z_{q N}^{(0)}:=\mathcal{Z}_{q}^{(0)}\{u=0\} \oslash_{q} N !_{q}
$$

где $q$-деформированный факториал $N !_{q}$ числа $N \gg 1$ определяется формулой Стирлинга (П.7) (см. приложение), которой удобно придать вид ${ }^{1)}$

$$
N !_{q} \simeq \exp _{q}\left[\frac{N}{|2-q|} \ln _{q}\left(N \oslash_{q} e_{q}\right)\right], \quad q \neq 2 .
$$

\footnotetext{
1) Отметим, что при определении деформированного произведения в работе [39] упускалось условие положительности выражения, стоящего в квадратных скобках в формулах (П.4) (см. приложение). Поэтому для больших чисел $n \gg 1 q$-логарифм $\ln _{q}\left(n !_{q}\right)$ в формуле (П.7) может принимать отрицательные значения при $q>2$. Во избежание этого следует брать разность $q-2$ по абсолютному значению.
} 
Здесь $e_{q} \equiv|2-q|^{1 /(1-q)}-q$-деформированное основание натурального логарифма, принимающее обычное значение $e_{1} \equiv e \simeq 2.718$ при $q=1$. Из формул (44), (П.7) (см. приложение) с использованием производящего функционала (41) находим

$$
Z_{q N}^{(0)}=\underbrace{z_{q}^{(0)}(0) \otimes_{q} \cdots \otimes_{q} z_{q}^{(0)}(0)}_{N} \oslash_{q} \exp _{q}\left[\frac{N}{|2-q|} \ln _{q}\left(N \oslash_{q} e_{q}\right)\right],
$$

где обозначено $z_{q}^{(0)}(0) \equiv z_{q}^{(0)}\left(u_{i}=0\right)$. Проводя $q$-логарифмирование, приводим равенство (45) к аддитивной форме $\ln _{q}\left(Z_{q N}^{(0)}\right)=N \ln _{q}\left(z_{q}^{(0)}\right)$, где одночастичная статистическая сумма определяется выражением

$$
z_{q}^{(0)}:=\exp _{q}\left\{\ln _{q}\left[z_{q}^{(0)}(0)\right]-\frac{1}{|2-q|} \ln _{q}\left(N \oslash_{q} e_{q}\right)\right\},
$$

в котором величина $z_{q}^{(0)}(0)$ задается равенством $(42)$.

Для вычисления удельной статистической суммы удобно ввести переменную

$$
y=\frac{|(1-q)(2-q)|}{2 \Delta^{2}} x^{2},
$$

переход к которой приводит к удвоению интеграла в формуле (42). В результате получаем выражение

$$
z_{q}^{(0)}=\exp _{q}\left\{\ln _{q}\left[Q \Delta B\left(Q_{0}, \frac{1}{2}\right)\right]-\frac{1}{|2-q|} \ln _{q}\left(N \oslash_{q} e_{q}\right)\right\},
$$

где бета-функция определена стандартным образом [40], а функции параметра неаддитивности $Q=Q(q)$ и $Q_{0}=Q_{0}(q)$ заданы равенствами

$$
Q=\sqrt{\frac{2}{|(1-q)(2-q)|}}, \quad Q_{0}= \begin{cases}\frac{1}{1-q}+1, & q \notin(1,2), \\ \frac{1}{q-1}-\frac{1}{2}, & q \in(1,2) .\end{cases}
$$

В явном виде $q$-логарифм статистической суммы (48) записывается следующим образом:

$$
\ln _{q}\left(z_{q}^{(0)}\right)=\frac{|2-q|\left[z_{q}^{(0)}(0)\right]^{1-q}-N^{1-q}}{(1-q)|2-q|}, \quad z_{q}^{(0)}(0)=Q \Delta B\left(Q_{0}, \frac{1}{2}\right) .
$$

Это выражение принимает скейлинговую форму $\ln _{q}\left(z_{q}^{(0)}\right) \propto N^{1-q}$ при условии $\Delta=$ $\Delta_{1} N$, где параметр $\Delta_{1}$ не зависит от числа частиц. Это условие подтверждается примером аддитивных систем, где для $d$-мерного идеального газа параметр $\Delta_{1}=$ $\left(m T /\left(2 \pi \hbar^{2}\right)\right)^{d / 2} n^{-1}$ определяется плотностью $n=N / V ; V$ - объем [37].

Моменты свободного поля определяются выражением

$$
\left\langle x^{m}\right\rangle_{q}^{(0)}:=\frac{1}{z_{q}^{(0)}(0)} \int_{-\infty}^{\infty} x^{m} e_{q}^{-x^{2} /[2]_{q} \Delta^{2}} d x .
$$

Для нечетных порядков $m=2 n-1, n=1,2, \ldots$, имеем $\left\langle x^{2 n-1}\right\rangle_{q}^{(0)}=0$, а моменты четных порядков $m=2 n, n=1,2, \ldots$, принимают вид

$$
\left\langle x^{2 n}\right\rangle_{q}^{(0)}=\frac{(Q \Delta)^{2 n+1}}{z_{q}^{(0)}(0)} B\left[Q_{0}(n), n+\frac{1}{2}\right], \quad Q_{0}(n)= \begin{cases}\frac{1}{1-q}+1, & q \notin(1,2) \\ \frac{1}{q-1}-\left(n+\frac{1}{2}\right), & q \in(1,2) .\end{cases}
$$


При учете ангармонизма $V(x)$ производящий функционал представляется деформированным произведением типа (41), где одночастичная составляющая имеет вид (ср. с формулой (42))

$$
z_{q}(u)=\int \exp _{q}\left\{\int\left[u x-\left(\frac{x^{2}}{[2]_{q} \Delta^{2}}+V(x)\right)\right] d \mathbf{r} d t\right\} \delta x .
$$

Проводя разложение экспоненты Цаллиса (П.2) (см. приложение) по степеням возмущения $V(x)$, находим $z_{q}(0)=z_{q}^{(0)}(0)+z_{q}^{\prime}(0)$, где добавка к гармоническому приближению представляется рядом теории возмущений:

$$
z_{q}^{\prime}(0)=Q \Delta \sum_{n=1}^{\infty}(-1)^{n} C(q, n) \int_{0}^{\infty} V^{n}(y)(1 \mp y)_{+}^{1 /(1-q)-n} y^{-1 / 2} d y
$$

с коэффициентами

$$
C(q, n)=(1-q)^{n} \prod_{m=1}^{n} \frac{(2-q) /(1-q)-m}{m} .
$$

При $q \notin(1,2)$ в биноме подынтегрального выражения (54) следует взять верхний знак, при котором ограничение, наложенное нижним индексом, сводит верхний предел интегрирования к единице; в интервале $q \in(1,2)$ берется нижний знак, и указанное ограничение снимается.

В первом порядке поправка $z_{q}^{\prime}(0)$ составляет

$$
z_{q}^{(1)}(0)=-Q \Delta \int_{0}^{\infty} V(y)(1 \mp y)_{+}^{q /(1-q)} y^{-1 / 2} d y .
$$

Соответственно во втором порядке имеем

$$
z_{q}^{(2)}(0)=\frac{Q q}{2} \Delta \int_{0}^{\infty} V^{2}(y)(1 \mp y)_{+}^{(2 q-1) /(1-q)} y^{-1 / 2} d y
$$

Для стандартного возмущения

$$
V=\frac{\lambda_{1}}{3} x^{3}+\frac{\mu_{1}}{4} x^{4}
$$

определенного параметрами $\lambda_{1}, \mu_{1}$, поправка первого порядка (56) не содержит кубического ангармонизма, поскольку он приводит к подынтегральной функции, антисимметричной относительно переменной $x$, от которой совершается переход к $y$ согласно (47). В результате получаем

$$
z_{q}^{(1)}(0)=-\frac{\mu\left(Q \Delta_{1}\right)^{5}}{4} B\left(Q_{1}, \frac{5}{2}\right) N, \quad \mu \equiv \mu_{1} N^{4}, \quad Q_{1}= \begin{cases}\frac{1}{1-q}, & q \notin(1,2), \\ \frac{1}{q-1}-\frac{3}{2}, & q \in(1,2) .\end{cases}
$$

Кубический ангармонизм проявляется только в слагаемом (57) второго порядка, где он дает вклад

$$
z_{q}^{(2)}(0)=\frac{\lambda^{2} q\left(Q \Delta_{1}\right)^{7}}{18} B\left(Q_{2}, \frac{7}{2}\right) N, \quad \lambda \equiv \lambda_{1} N^{3}, \quad Q_{2}= \begin{cases}\frac{1}{1-q}-1, & q \notin(1,2), \\ \frac{1}{q-1}-\frac{3}{2}, & q \in(1,2) .\end{cases}
$$


Подстановка поправок (59), (60) в квадратные скобки выражения (50) приводит к уточнению величины одночастичной статистической суммы $z_{q}$.

С учетом возмущения $V(x)$ момент порядка $m$ определяется выражением (ср. с формулой (51))

$$
\left\langle x^{m}\right\rangle_{q}=\frac{(Q \Delta)^{m+1}}{z_{q}(0)} \int_{0}^{\infty}[(1 \mp y)-(1-q) V(y)]_{+}^{1 /(1-q)} y^{(m-1) / 2} d y .
$$

Подобно (54) здесь при $q \notin(1,2)$ следует взять верхний знак, при котором верхний предел интегрирования сводится к единице; в интервале $q \in(1,2)$ берется нижний знак. Далее в подынтегральном выражении (61) следует провести разложение по $V(x)$ и учесть подобную поправку (54) для производящей функции $z_{q}(0)=z_{q}^{(0)}(0)+z_{q}^{\prime}(0)$. В результате изменение момента $\left\langle x^{m}\right\rangle_{q}^{\prime}=\left\langle x^{m}\right\rangle_{q}-\left\langle x^{m}\right\rangle_{q}^{(0)}$ принимает вид

$$
\begin{aligned}
\left\langle x^{m}\right\rangle_{q}^{\prime}= & -\frac{z_{q}^{\prime}(0)}{z_{q}^{(0)}(0)}\left\langle x^{m}\right\rangle_{q}^{(0)}+\frac{(Q \Delta)^{m+1}}{z_{q}^{(0)}(0)} \sum_{n=1}^{\infty}(-1)^{n} C(q, n) \times \\
& \times \int_{0}^{\infty} V^{n}(y)(1 \mp y)_{+}^{1 /(1-q)-n} y^{(m-1) / 2} d y,
\end{aligned}
$$

где в отличие от формулы (54) последний множитель содержит степень $(m-1) / 2$ вместо -1/2. Комбинируя выражение (62) с равенствами (48), (52) и (54), находим

$$
\begin{aligned}
\left\langle x^{m}\right\rangle_{q}^{\prime}= & \frac{(Q \Delta)^{m+1}}{z_{q}^{(0)}(0)} \sum_{n=1}^{\infty}(-1)^{n} C(q, n) \int_{0}^{\infty} V^{n}(y)(1 \mp y)_{+}^{1 /(1-q)-n} \times \\
& \times\left\{y^{m / 2}-\frac{Q \Delta}{z_{q}^{(0)}} B\left[Q_{0}\left(\frac{m}{2}\right), \frac{m+1}{2}\right]\right\} y^{-1 / 2} d y .
\end{aligned}
$$

Как показывает пример определения поправок (59), (60), в силу связи (47) слагаемые ряда (63), содержащие множитель $y^{(m-1) / 2}$ с нечетным $m$, дают нулевой вклад. С учетом формулы (58) поправка первого порядка принимает вид

$$
\begin{aligned}
\left\langle x^{m}\right\rangle_{q}^{(1)}= & -\frac{(Q \Delta)^{m+1}}{z_{q}^{(0)}(0)} \int_{0}^{\infty}(1 \mp y)_{+}^{q /(1-q)}\left(\frac{\lambda}{3} y^{3 / 2}+\frac{\mu}{4} y^{2}\right) \times \\
& \times\left\{y^{m / 2}-\frac{Q \Delta}{z_{q}^{(0)}} B\left[Q_{0}\left(\frac{m}{2}\right), \frac{m+1}{2}\right]\right\} y^{-1 / 2} d y .
\end{aligned}
$$

Опуская антисимметричные подынтегральные слагаемые, при нечетных показателях $m=2 n-1$ находим

$$
\left\langle x^{2 n-1}\right\rangle_{q}^{(1)}=-\frac{(Q \Delta)^{2 n}}{z_{q}^{(0)}(0)} \int_{0}^{\infty}(1 \mp y)_{+}^{q /(1-q)}\left\{\frac{\lambda}{3} y^{n+1 / 2}-\frac{\mu}{4} \frac{Q \Delta}{z_{q}^{(0)}} B\left[Q_{0}\left(n-\frac{1}{2}\right), n\right] y^{3 / 2}\right\} d y .
$$

Отсюда окончательно следует

$$
\left\langle x^{2 n-1}\right\rangle_{q}^{(1)}=\frac{(Q \Delta)^{2 n}}{z_{q}^{(0)}(0)}\left\{\frac{\mu}{4} \frac{Q \Delta B\left[Q_{\text {odd }}(1), 5 / 2\right]}{z_{q}^{(0)}} B\left[Q_{0}\left(n-\frac{1}{2}\right), n\right]-\frac{\lambda}{3} B\left[Q_{\text {odd }}(n), n+\frac{3}{2}\right]\right\},
$$

где обозначено

$$
Q_{\text {odd }}(n)= \begin{cases}\frac{1}{1-q}, & q \notin(1,2), \\ \frac{1}{q-1}-\left(n+\frac{1}{2}\right), & q \in(1,2) .\end{cases}
$$


Соответственно моменты четного порядка $m=2 n$ записываются в форме

$$
\left\langle x^{2 n}\right\rangle_{q}^{(1)}=-\frac{\mu}{4} \frac{(Q \Delta)^{2 n+1}}{z_{q}^{(0)}(0)} \int_{0}^{\infty}(1 \mp y)_{+}^{q /(1-q)}\left\{y^{n+3 / 2}-\frac{Q \Delta}{z_{q}^{(0)}} B\left[Q_{0}(n), n+\frac{1}{2}\right] y^{3 / 2}\right\} d y .
$$

В результате получаем

$$
\left\langle x^{2 n}\right\rangle_{q}^{(1)}=\frac{\mu}{4} \frac{(Q \Delta)^{2 n+1}}{z_{q}^{(0)}(0)}\left\{\frac{Q \Delta B\left[Q_{\mathrm{even}}(0), 5 / 2\right]}{z_{q}^{(0)}} B\left[Q_{0}(n), n+\frac{1}{2}\right]-B\left[Q_{\mathrm{even}}(n), n+\frac{5}{2}\right]\right\},
$$

где

$$
Q_{\text {even }}(n)= \begin{cases}\frac{1}{1-q}, & q \notin(1,2), \\ \frac{1}{q-1}-\left(n+\frac{3}{2}\right), & q \in(1,2) .\end{cases}
$$

Как показывают выражения (66), (69), поправки к статистической сумме (54) приводят к положительным вкладам в моменты (61). С другой стороны, в моменты нечетного порядка (66) дают вклады как кубический, так и биквадратный ангармонизмы, тогда как при четном порядке сказывается только последний.

Везде выше мы пренебрегали вкладом градиентных слагаемых и межчастичным взаимодействием, благодаря чему наиболее удобным было использование одноузельного приближения. Для учета указанных эффектов следует перейти к волновому представлению, в рамках которого лагранжиан свободного неоднородного поля принимает вид (ср. с формулой (40))

$$
\mathcal{L}_{q}^{(0)}:=\frac{2}{[2]_{q}} \sum_{\mathbf{k}}\left(\frac{1}{\Delta^{2}}+\alpha \mathbf{k}^{2}\right)\left|x_{\mathbf{k}}\right|^{2}
$$

где $\alpha>0$ - параметр неоднородности. Соответственно двухчастичное взаимодействие определяется вкладом

$$
W_{q}:=\frac{2}{[4]_{q} N} \sum_{\mathbf{k}, \mathbf{k}^{\prime}, \mathbf{k}^{\prime \prime}} w_{\mathbf{k}-\mathbf{k}^{\prime}} x_{\mathbf{k}^{\prime}}^{*} x_{-\mathbf{k}^{\prime}+\mathbf{k}^{\prime \prime}}^{*} x_{-\mathbf{k}+\mathbf{k}^{\prime \prime}} x_{\mathbf{k}},
$$

интенсивность которого задается ядром $w_{\mathbf{k}-\mathbf{k}^{\prime}}$ при $x_{\mathbf{k}}^{*}=x_{-\mathbf{k}}$.

В рамках волнового представления поведение системы определяется $N$ степенями свободы, интенсивности которых задаются величинами $x_{\mathbf{k}}$. Статистическая сумма каждой из этих мод задается теми же формулами (50), (54), (56), (57), (59) и (60), в которых, как показывает сравнение равенств (71) и (40), следует заменить $\Delta$ на $\Delta / \sqrt{1+\alpha \Delta^{2} \mathbf{k}^{2}}$; такую же замену следует провести и в равенствах $(52)$, (61)-(66), (68) и (69), определяющих моменты наблюдаемых величин. Что касается межчастичного взаимодействия, то наиболее простым образом его учет достигается в приближении среднего поля, в рамках которого в равенстве (72) следует пренебречь суммой по волновому вектору $\mathbf{k}^{\prime \prime}$, ядро $w_{\mathbf{k}-\mathbf{k}^{\prime}}$ положить равным постоянному значению $w_{\mathbf{0}}$, отвечающему $\mathbf{k}=\mathbf{k}^{\prime}$, а произведения типа $x_{\mathbf{k}}^{*} x_{-\mathbf{k}}$ заменить средним значением $\rho \equiv N^{-1} \sum_{\mathbf{k}}\left\langle x_{\mathbf{k}}^{*} x_{-\mathbf{k}}\right\rangle$. В результате выражение (72) принимает квадратичную форму

$$
W_{q} \simeq \frac{4 w_{\mathbf{0}} \rho}{[4]_{q}} \sum_{\mathbf{k}} x_{\mathbf{k}}^{*} x_{-\mathbf{k}}
$$

подобную выражению (71). 
Таким образом, учет пространственной неоднородности (71) и межчастичного взаимодействия (72) оставляет неизменной форму равенств (50), (52), (54), (56), $(57),(59)-(66),(68)$ и (69), определяющих статистическую сумму и моменты наблюдаемых величин для каждой из степеней свободы, отвечающих волновому вектору $\mathbf{k}$. При этом в указанных равенствах под параметром $\Delta$ следует понимать диспергирующее значение

$$
\Delta_{\mathbf{k}}=\frac{\Delta}{\sqrt{\left(1+\left(2[2]_{q} /[4]_{q}\right) \Delta^{2} w_{\mathbf{0}} \rho\right)+\alpha \Delta^{2} \mathbf{k}^{2}}} .
$$

С учетом аддитивности $q$-логарифмов статистических сумм $z_{q \mathbf{k}}$, приходящихся на каждую степень свободы $\mathbf{k}$, полное значение $Z_{q N}$ для $N$ частиц определяется равенством

$$
\ln _{q}\left(Z_{q N}\right)=\sum_{\mathbf{k}} \ln _{q}\left(z_{q \mathbf{k}}\right) .
$$

\section{6. ОБСУЖДЕНИЕ РЕЗУЛЬТАТОВ}

Проведенное рассмотрение показывает, что деформация статистического распределения, приводящая к неаддитивности термодинамических потенциалов, не требует принципиальных изменений при использовании квантово-полевых методов [29]-[31] для описания сложных систем. В частности, оказывается, что эволюция наиболее вероятных значений параметра порядка и амплитуды его флуктуаций вообще не испытывает изменений, тогда как вероятности реализации различных фазовых траекторий зависят от параметра неаддитивности. При этом полевой формализм, основанный на методе производящего функционала [31], модифицируется в том же духе, что и статистика Цаллиса [3].

Термодинамические свойства неаддитивной системы, состоящей из $N$ частиц, определяются $q$-деформированной свободной энергией $F_{q N}:=-T \ln _{q}\left(Z_{q N}\right)$. Поскольку $q$-логарифм статистической суммы сохраняет свою аддитивность, то определенный таким образом термодинамический потенциал может быть представлен в стандартной форме $F_{q N}=N f_{q}$, где удельная свободная энергия $f_{q}:=-T \ln _{q}\left(z_{q}\right)$ задается статистической суммой $z_{q}$, приходящейся на частицу. При $q \neq 1$ величина $z_{q}$ зависит от числа частиц $N$, благодаря чему нарушается условие аддитивности $F_{q N} \propto N$ полной свободной энергии.

Следуя рассмотрению, проведенному в предыдущем разделе, представим зависимость удельной статистической суммы $z_{q}$ от параметра неаддитивности $q$. Из рис. 4 видно, что в гармоническом приближении с ростом $q$ в интервале $q \in(0,2)$ величина $\ln _{q}\left(z_{q}^{(0)}\right)$ монотонно возрастает от конечного значения $\ln _{0}\left(z_{0}^{(0)}\right)=\left((4 / 3) \Delta_{1}-1 / 2\right) N$ до бесконечности (формулы (43), (44) показывают, что расходимость $q$-логарифма статистической суммы при $q=2$ обусловлена бесконечным возрастанием $q$-факториала $N !_{q}$, учитывающего тождественность частиц). При этом реализуются асимптотики

$N^{q-1} \ln _{q}\left(z_{q}^{(0)}\right) \simeq \begin{cases}{\left[\ln \left(\sqrt{2 \pi} \Delta_{1}\right)+\frac{1}{|2-q|}\right]+\left[\frac{7}{8}-\frac{1}{2} \ln ^{2}\left(\sqrt{2 \pi} \Delta_{1}\right)\right](q-1),} & |q-1| \ll 1 ; \\ \frac{1}{|2-q|}, & |2-q| \ll 1 .\end{cases}$

Согласно рис. 4 ангармонизм очень слабо влияет на статистическую сумму свободного поля. 


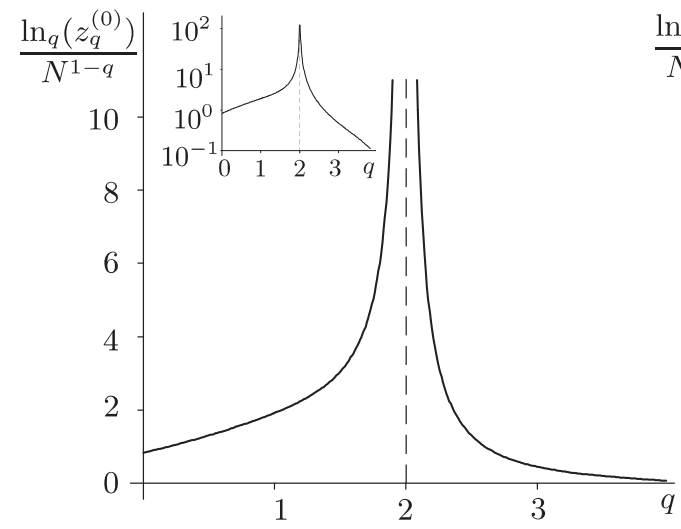

a

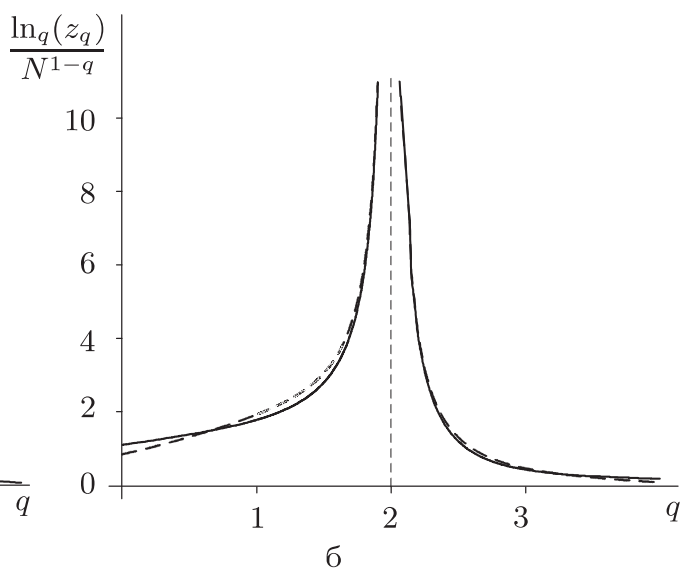

б

Рис. 4. Зависимость $q$-логарифма одночастичной статистической суммы (50) от параметра неаддитивности, на врезке использованы полулогарифмические координаты (а); та же зависимость для статистической суммы (б) в случае свободного поля (штриховая линия) и с учетом ангармонизма при $\lambda=\mu=12$ (сплошная).

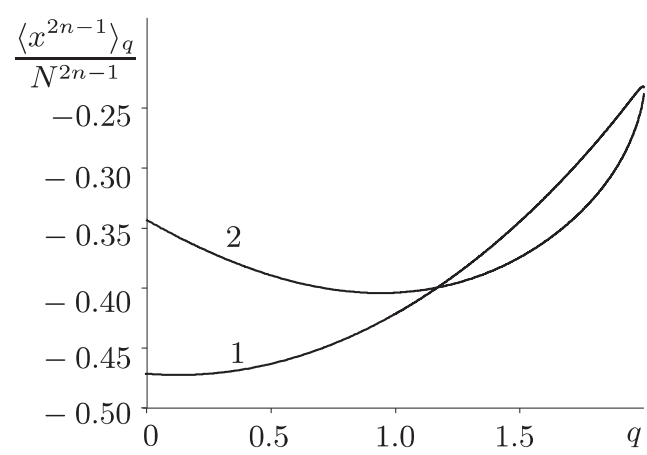

a

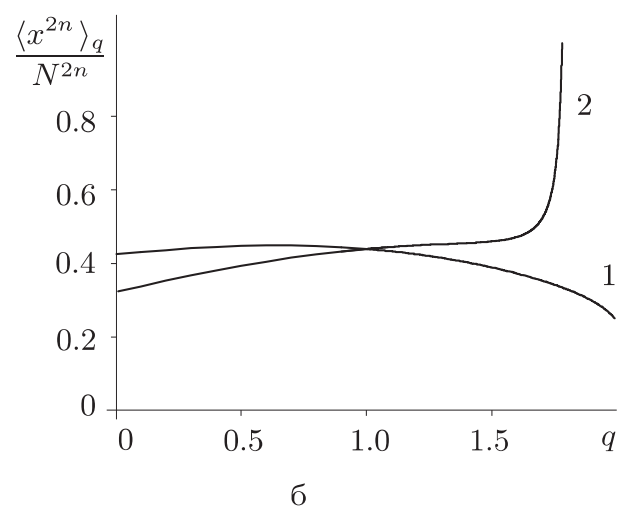

Рис. 5. Зависимость моментов $\left\langle x^{2 n-1}\right\rangle_{q}$ (а) и $\left\langle x^{2 n}\right\rangle_{q}$ (б) от параметра неад-
дитивности при $\lambda, \mu=12$ (кривые 1,2 отвечают $n=1,2$ соответственно).

Представим поведение моментов наблюдаемых величин в зависимости от параметра неаддитивности. Поскольку для свободного поля моменты нечетных порядков равны нулю, то на рис. 5 приведены данные для больших значений параметров ангармонизма $\lambda=\mu=12$. Обращает на себя внимание тот факт, что при фиксированном порядке $m$ момент $\left\langle x^{m}\right\rangle_{q}$ масштабируется числом частиц $N^{m}$, взятым в той же степени. Кроме того, оказывается, что моменты $\left\langle x^{2 n-1}\right\rangle_{q}$ нечетного порядка принимают отрицательные значения, а $\left\langle x^{2 n}\right\rangle_{q}$ - положительные. Из сравнения кривых, отвечающих различным степеням ангармонизма, на рис. 6 видно, что его рост приводит к увеличению абсолютных значений моментов нечетного порядка, тогда как для четных ангармонизм размывает особенность, наблюдающуюся для моментов $\left\langle x^{2 n}\right\rangle_{q}^{(0)}$ свободного поля при $q=2$. Подобно удельной статистической сумме (76) 


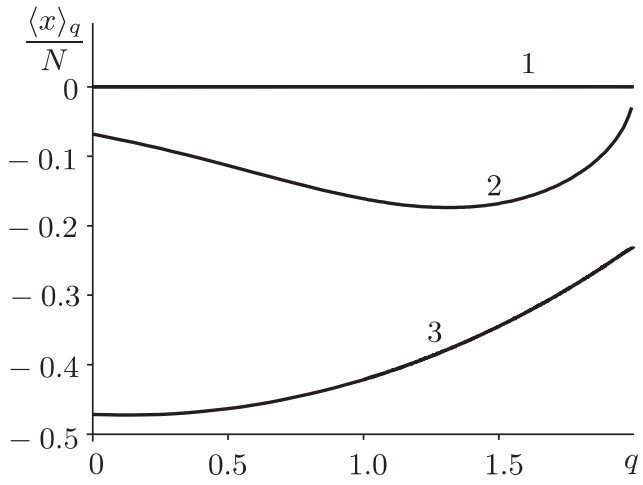

a

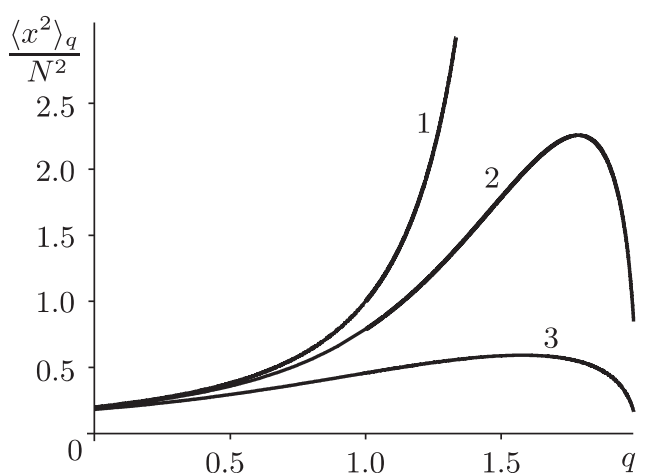

б

Рис. 6. Зависимость среднего значения $\langle x\rangle_{q}$ от параметра неаддитивности и ангармонизма: кривые $1,2,3$ отвечают $\lambda, \mu=0,1,12$ соответственно (а); та же зависимость для дисперсии $\left\langle x^{2}\right\rangle_{q}$ : кривые $1,2,3$ отвечают $\lambda, \mu=0.0,0.1,1.0$ соответственно (б).

с ростом параметра неаддитивности $q$ в интервале $q \in(0,2)$ четные моменты $\left\langle x^{2 n}\right\rangle_{q}^{(0)}$ монотонно возрастают от конечного значения $\left\langle x^{2 n}\right\rangle_{0}^{(0)}=3 \Delta_{1}^{2 n} N^{2 n} /(2 n+1)(2 n+3)$ до бесконечности. При этом вблизи значений $q=1$ и $q=2$ реализуются асимптотики

$$
\frac{\left\langle x^{2 n}\right\rangle_{q}^{(0)}}{N^{2 n}} \simeq \begin{cases}\frac{(2 n) !}{n !}\left(\frac{\Delta_{1}^{2}}{2}\right)^{n}\left[1+\frac{1}{2}\left(n+\frac{1}{2}\right)\left(n+\frac{3}{2}\right)(q-1)\right], & |q-1| \ll 1, \\ \frac{(2 n) !}{n !}\left(\frac{\Delta_{1}^{2}}{2}\right)^{n}|2-q|^{-n}, & |2-q| \ll 1 .\end{cases}
$$

При интерпретации найденных зависимостей статистической суммы и моментов наблюдаемых величин от параметра неаддитивности следует иметь в виду, что в самоподобных системах его значение $q$ не является свободным. Это связано с тем, что при деформации фазового пространства, растягивающей одну из осей $x, p$ в $\lambda$ раз и сжимающей другую в той же степени (в результате фазовый объем не изменяется), должно выполняться условие самоподобия распределения состояний $x$, p. Это выражается в том, что физические значения величин задаются не исходной вероятностью $p(x)$, а эскортной (см. формулу (П.1)), представляющей степенную функцию $p(x)$. Исследование условий самоподобия показывает, что их выполнение обеспечивается уравнением [41]

$$
\left(\lambda^{q}-1\right)\left(\lambda^{q-1}-1\right)=(\lambda-1)^{2},
$$

определяющим связь параметра неаддитивности $q$ и деформации $\lambda$. В результате получаем зависимость, приведенную на рис. 7.

Этот рисунок показывает, что изменение деформации $\lambda$ от нуля до единицы приводит к спаданию параметра неаддитивности от $q=2$ до золотого сечения $q=1.618$, а при дальнейшем росте $\lambda$ до бесконечности параметр $q$ медленно уменьшается до $q=1.5$. Согласно рис. $4-6$ при этом удельная статистическая сумма также уменьшается, а моменты наблюдаемых величин могут изменяться немонотонным образом. 


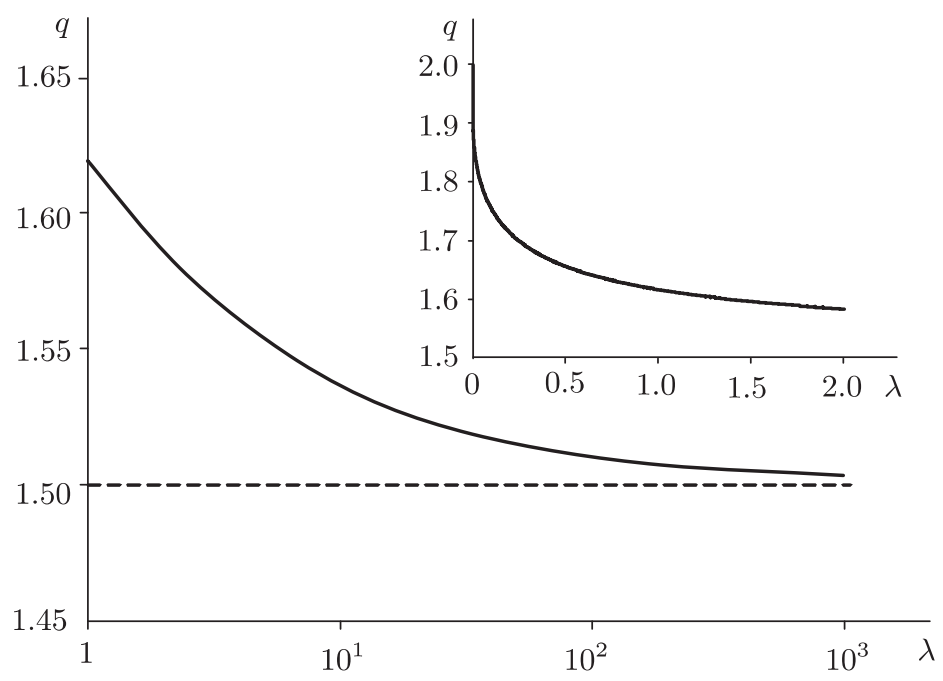

Рис. 7. Зависимость параметра неаддитивности $q$ от деформации $\lambda$ самоподобной системы.

ПРИЛОЖЕНИЕ

\section{Формализм неаддитивной статистической системы}

В отличие от обычного статистического ансамбля неаддитивная система подчиняется статистике Цаллиса [3], [38], в рамках которой состояния распределены не по Гиббсу, а согласно эскортной вероятности [7]

$$
P_{q}(x)=\frac{p^{q}(x)}{\int p^{q}(x) d x}, \quad p(x)=Z_{q}^{-1} e_{q}^{x},
$$

где статистическая сумма $Z_{q}$ определяется условием нормировки исходной вероятности $p(x)$. Последняя, в свою очередь, задается деформированной экспонентой Цаллиса

$$
e_{q}^{x}:=[1+(1-q) x]_{+}^{1 /(1-q)}, \quad[y]_{+} \equiv \max (y, 0),
$$

которая в пределе $q \rightarrow 1$ сводится к обычной экспоненте $e^{x}=e_{1}^{x}$. Соответственно логарифм Цаллиса, играющий роль функции, обратной экспоненте (П.2), определяется равенством

$$
\ln _{q}(x) \equiv \frac{x^{1-q}-1}{1-q}
$$

Кроме того, сумма, разность, произведение и частное положительных величин $x, y$ принимают вид [35]

$$
\begin{gathered}
x \oplus_{q} y:=x+y+(1-q) x y, \quad x \ominus_{q} y:=\frac{x-y}{1+(1-q) y}, \\
x \otimes_{q} y:=\left[x^{1-q}+y^{1-q}-1\right]_{+}^{1 /(1-q)}, \quad x \oslash_{q} y:=\left[x^{1-q}-y^{1-q}+1\right]_{+}^{1 /(1-q)} .
\end{gathered}
$$


При этом функции (П.2), (П.3) удовлетворяют правилам

$$
\begin{aligned}
\ln _{q}\left(x \otimes_{q} y\right)=\ln _{q} x+\ln _{q} y, & \ln _{q}\left(x \oslash_{q} y\right)=\ln _{q} x-\ln _{q} y, \\
e_{q}^{x} \otimes_{q} e_{q}^{y}=e_{q}^{x+y}, & e_{q}^{x} \oslash_{q} e_{q}^{y}=e_{q}^{x-y}, \\
\ln _{q}(x y)=\ln _{q} x \oplus_{q} \ln _{q} y, & \ln _{q}\left(\frac{x}{y}\right)=\ln _{q} x \ominus_{q} \ln _{q} y \\
e_{q}^{x} e_{q}^{y}=e_{q}^{x \oplus_{q} y}, & \frac{e_{q}^{x}}{e_{q}^{y}}=e_{q}^{x \ominus_{q} y}
\end{aligned}
$$

а $q$-факториал

$$
n !_{q}:=1 \otimes_{q} 2 \otimes_{q} \cdots \otimes_{q} n
$$

натурального числа $n \gg 1$ выражается формулой Стирлинга [39]

$$
\ln _{q}\left(n !_{q}\right) \simeq \begin{cases}\left(\frac{n}{2-q}+\frac{1}{2}\right) \ln _{q} n-\frac{n-1}{2-q}, & 0<q \neq 2, \\ {\left[n-\frac{1}{2}\left(1+\frac{1}{n}\right)\right]-\ln n,} & q=2 .\end{cases}
$$

Фурье-образ функции $f(x)$ определяется равенствами

$$
F_{q}[f](k):=\int_{-\infty}^{\infty} f(x) \otimes_{q} e_{q}^{i k x} d x=\int_{-\infty}^{\infty} e_{q}^{i k x+\ln _{q}(f(x))} d x,
$$

в первом из которых использовано деформированное произведение, а во втором правила (П.5). С учетом (П.2), (П.4) подынтегральное выражение в первом из равенств (П.8) переписывается следующим образом:

$$
\begin{aligned}
e_{q}^{i k x} \otimes_{q} f(x) & =\left\{[1+(1-q) i k x]+[f(x)]^{1-q}-1\right\}_{+}^{1 /(1-q)}= \\
& =f(x)\left\{1+(1-q) i k x[f(x)]^{q-1}\right\}_{+}^{1 /(1-q)}=f(x) e_{q}^{i k x[f(x)]^{q-1}} .
\end{aligned}
$$

В результате приходим к выражению [42]

$$
F_{q}[f](k)=\int_{-\infty}^{\infty} f(x) e_{q}^{i k x[f(x)]^{q-1}} d x
$$

где переход от деформированного произведения к обычному достигается за счет введения степени исходной функции $f(x)$ в показатель экспоненты. При этом $\delta$-функция представляется равенством [43]

$$
\delta_{q}(x)=\frac{2 \pi}{2-q} \int_{-\infty}^{\infty} e_{q}^{i k x} d k, \quad q \in[1,2] .
$$

\section{Список литературы}

[1] Р. Балеску, Равновесная и неравновесная статистическая механика, Мир, М., 1978.

[2] А.И. Олемской, Синергетика сложных систем: Феноменология и статистическая теория, КРАСАНД, М., 2009.

[3] C. Tsallis, Introduction to Nonextensive Statistical Mechanics. Approaching a Complex World, Springer, New York, 2009.

[4] C. Tsallis, J. Stat. Phys., 52:1-2 (1988), 479-487.

[5] E. M. Curado, C. Tsallis, J. Phys. A, 24:2 (1991), L69-L72. 
[6] S. Abe, Phys. Lett. A, 224:6 (1997), 326-330.

[7] C. Tsallis, R.S. Mendes, A. R. Plastino, Physica A, 261:3-4 (1998), 534-554.

[8] G. Kaniadakis, Phys. Rev. E, 66:5 (2002), 056125, 17 pp., arXiv: cond-mat/0210467.

[9] G. Kaniadakis, M. Lissia, A. M. Scarfone, Phys. A, 340:1-3 (2004), 41-49; Phys. Rev. E, 71:4 (2005), 046128, 12 pp., arXiv: cond-mat/0409683.

[10] J. Naudts, Phys. A, 340:1-3 (2004), 32-40.

[11] A. M. Scarfone, T. Wada, Phys. Rev. E, 72:2 (2005), 026123, 13 pp., arXiv: cond-mat/0504117.

[12] A. Lavagno, A. M. Scarfone, N. P. Swamy, Eur. Phys. J. B, 50:1-2 (2006), 351-354, arXiv: cond-mat/0509477.

[13] A. Lavagno, A. M. Scarfone, N. P. Swamy, Phys. A, 40:30 (2007), 8635-8654, arXiv: 0706.0426.

[14] G. Vitiello, Topological defects, fractals and the structure of quantum field theory, arXiv: 0807.2164.

[15] F. Wilczek, Fractional statistics and anyon superconductivity, World Sci., Singapore, 1990.

[16] L. C. Biedenharn, Phys. A, 22:18 (1989), L873-L878.

[17] A. J. Macfarlane, J. Phys. A, 22:21 (1989), 4581-4588.

[18] E. Celeghini, M. Rasetti, G. Vitiello, Phys. Rev. Lett., 66:16 (1991), 2056-2059.

[19] E. Heine, J. reine angew. Math., 32 (1846), 210-212; 34 (1847), 285-328.

[20] F. H. Jackson, Amer. J. Math., 38 (1909), 26; Mess. Math., 38 (1909), 57.

[21] H. Exton, q-Hypergeometric Functions and Applications, Ellis Horwood, New York, 1983.

[22] C. Kassel, Quantum Groups, Graduate Texts in Mathematics, 155, Springer, New York, 1995.

[23] A. Lavagno, N. P. Swamy, Phys. Rev. E, 61:2 (2000), 1218-1226, arXiv: quant-ph/9912111; 65:3 (2002), 036101, 5 pp.

[24] D. Sornette, Critical Phenomena in Natural Sciences. Chaos, Fractals, Selforganization and Disorder: Concepts and Tools, Springer, Berlin, 2006.

[25] A. Erzan, J.-P. Eckmann, Phys. Rev. Lett., 78:17 (1997), 3245-3248.

[26] A. Erzan, Phys. Lett. A, 225:4-6 (1997), 235-238.

[27] A. B. Adiba, A. A. Moreirab, J.S. Andrade Jr., M.P. Almeida, Phys. A, 322 (2003), $276-284$.

[28] S. Abe, Y. Okamoto (eds.), Nonextensive Statistical Mechanics and its Applications, Papers from the IMS Winter School on Statistical Mechanics: Nonextensive Generalization of Boltzmann-Gibbs Statistical Mechanics and its Applications (Okazaki, February 15-18, 1999), Lecture Notes in Physics, 560, Springer, Berlin, 2001.

[29] А. А. Абрикосов, А. П. Горьков, И. Е. Дзялошинский, Методы квантовой теории поля в статистической физике, ГИФМЛ, М., 1962.

[30] Е. М. Лифшиц, Л. П. Питаевский, Статистическая физика. Ч. 2. Теория конденсированного состояния, Физматлит, М., 2002.

[31] J. Zinn-Justin, Quantum Field Theory and Critical Phenomena, Clarendon Press, Oxford, 1993.

[32] A. I. Olemskoi, S. S. Borysov, I. A. Shuda, Eur. Phys. J. B, 77:2 (2010), 219-231.

[33] A. I. Olemskoi, I. A. Shuda, Phys. Lett. A, 373:44 (2009), 4012-4016.

[34] P. C. Martin, E. D. Siggia, H. A. Rose, Phys. Rev. A, 8:1 (1973), 423-437.

[35] E. P. Borges, Phys. A, 340:1-3 (2004), 95-101, arXiv: cond-mat/0304545.

[36] H. Risken, The Fokker-Planck Equation. Methods of Solution and Applications, Springer, Berlin, 1984.

[37] Л. Д. Ландау, Е. М. Лифшиц, Статистическая физика, Ч. 1, Физматлит, М., 2002.

[38] Ю. Г. Рудой, ТМФ, 135:1 (2003), 3-54. 
[39] H. Suyari, q-Stirling's formula in Tsallis statistics, arXiv: cond-mat/0401541.

[40] М. Абрамовиц, И. Стиган (ред.), Справочник по специальным функциям с формулами, графиками и математическими таблицами, Наука, М., 1979.

[41] A. I. Olemskoi, A.S. Vaylenko, I. A. Shuda, Phys. A, 388:9 (2009), 1929-1938, arXiv: 0810.1189 .

[42] S. Umarov, C. Tsallis, S. Steinberg, Milan. J. Math., 76 (2008), 307-308.

[43] M. Jauregui, C. Tsallis, J. Math. Phys., 51 (2010), 063304.

Поступила в редакцию 31.08.2012 